\title{
On Lattice Points in Polyhedral Cross-Sections
}

\author{
Nickolay M. Korneenko and Nickolay N. Metelskij \\ Institute of Mathematics, Byelarus Academy of Sciences, \\ CIS 220072, Byelarus, Minsk, Surganova 11 \\ imanb@adonis.ias.msk.su
}

Communicated by Jörg M. Wills

\begin{abstract}
We prove that the convex hull of any $k^{d}+1$ points of a $d$-dimensional lattice contains $k+1$ collinear lattice points. (b) For a convex polyhedron we consider the numbers of its lattice points in consecutive parallel lattice hyperplanes (levels). We prove that if a polyhedron spans no more than $2^{d-1}$ levels, then this string of numbers may be arbitrary. On the other hand, we give an example of a string of $2^{d-1}+1$ numbers to which no convex polyhedron corresponds in $R^{d}$.
\end{abstract}

\section{Preliminaries}

Problems of counting lattice points in polytopes have gained considerable attention (see, e.g., [GW] and [S]) due to their relevance to integer programming and computer algebra. This paper gives some insight into possible arrangements of lattice points in linear and hyperplanar cross-sections of convex polyhedra. Earlier considerations of the problems discussed may be found in [KM].

Consider the space $R^{d}$ with its unit vectors $e^{1}, \ldots, e^{d} . Z$ is the common notation for the set of integers. For a set $S$ of $R^{d}$ define $Z(S)$ to be the set of its integer points.

A lattice $L$ in $R^{d}$ is a discrete subset of $R^{d}$ such that there exists a basis $\left\{v^{1}, \ldots, v^{1}\right\}$ of $R^{d}$ and

$$
L=\left\{\sum_{i=1}^{d} k_{i} v^{i}, k_{1}, \ldots, k_{d} \in Z\right\}
$$

In what follows, we consider the lattice to be fixed and lattice point coordinates will be given with respect to the corresponding basis.

Conv $(S)$ denotes the convex hull of $S$. The point set $S$ is said to be convexly independent if all points of $S$ are distinct extremal points (vertices) of $\operatorname{conv}(S)$. 


\section{Collinear Lattice Points in Convex Hulls of Lattice Point Sets}

We start with a theorem which is just a special case of Theorem 2, but we would like to consider it separately due to its simple elegancy and for other reasons stated below.

Theorem 1. The convex hull of $2^{d}+1$ distinct lattice points in $R^{d}$ contains at least three distinct collinear lattice points.

Proof. For a lattice point $X=\left(x_{1}, \ldots, x_{d}\right)$ consider its coordinates modulo 2 , i.e., let $\left(i_{1}, \ldots, i_{d}\right)$ be a $(0,1)$-valued vector such that $x_{j} \equiv i_{j}(\bmod 2), j=1, \ldots, d$. Clearly, there are only $2^{d}$ such distinct $(0,1)$-vectors. By the pigeonhole principle, among the $2^{d}+1$ given points there are two of them, say, $P$ and $Q$, with coinciding modulo 2 coordinates. The midpoint $M=(P+Q) / 2$ has integer coordinates, and the points $P, M$, and $Q$ are the required collinear ones.

Corollary 1. The convex hull of $2^{d}+1$ convexly independent lattice points in $R^{d}$ contains at least $2^{d}+2$ distinct lattice points.

There are two possible extensions of Theorem 1.

Theorem 2. The convex hull of $k^{d}+1$ distinct lattice points in $R^{d}$ contains at least $k+1$ distinct collinear lattice points.

Proof. Consider lattice point coordinates modulo $k$ and proceed as in the proof of Theorem 1: There are only $k^{d}$ possibilities, hence, there exist two given points $P$ and $Q$ such that $Q=P+k * T$, where $T$ is a nonzero lattice vector. Hence, $P$, $P+T, P+2 T, \ldots, P+k * T$ are the required collinear lattice points.

Similarly to Corollary 1 , we might state

Corollary 2. The convex hull of $k^{d}+1$ convexly independent lattice points in $R^{d}$ contains at least $k^{d}+k$ distinct lattice points.

However, Corollary 2 gives a rather weak estimate in contrast to Corollary 1 and this is the first reason to consider Theorem 1 separately. The second reason is that Theorem 1 seems to admit another way of extension.

Hypothesis 1. For any positive $k \leq d$, the convex hull of $2^{d}+1$ distinct lattice points in $R^{d}$ contains at least $2^{k}+1$ distinct lattice points in some $k$-dimensional plane.

Note. Theorem 1 is in a sense the best possible, for there do exist $2^{d}$ lattice points (namely, the vertices of the unit hypercube) with no three on a common line. The same is true for Theorem 2. 
A less graceful but more tight extension of Corollary 1 is

Corollary 3. The convex hull of $k^{d}+1$ distinct lattice points in $R^{d}$ with no $k$ of them on a common line contains at least $k^{d}+k$ distinct lattice points.

\section{Lattice Points in Consecutive Levels of a Convex Polyhedron}

The exposition in this section is in terms of the integer lattice, for convenience. However, all arguments are of an affine character, therefore the results remain valid for an arbitrary lattice in $R^{d}$.

Let $p$ be a bounded convex polyhedron in $R^{d}$. Consider the sequence of numbers of integer points in the cross-sections of $Z(P)$ by hyperplanes $x^{1}=i$ for all $i$. Let $\left\langle a_{1}, \ldots, a_{k}\right\rangle$ be its contiguous substring such that $a_{1}$ and $\mathrm{a}_{\mathrm{k}}$ are the first and the last nonzero elements. The corresponding cross-sections are called the levels of $P$. The strings $\left\langle a_{1}, \ldots, a_{k}\right\rangle$ have been considered in $[\mathrm{M}]$ and [KM] for defining equivalence classes on the set of all integer linear programs.

A string of nonnegative integers is called $d$-polyhedral if there is a convex polyhedron in $R^{d}$ generating it in the way described above.

A natural problem is to characterize the set of $d$-polyhedral strings. We give a partial answer here.

Lemma 1. If strings $a=\left\langle a_{1}, \ldots, a_{k}\right\rangle$ and $b=\left\langle b_{1}, \ldots, b_{m}\right\rangle$ are d-polyhedral, then their concatenation

$$
\left\langle a_{1}, \ldots, a_{k}, b_{1}, \ldots, b_{m}\right\rangle
$$

is $(d+1)$-polyhedral.

Proof. Let polyhedra $A$ and $B$ in $R^{d}$ generate strings $a$ and $\mathrm{b}$, respectively, and $R^{d}$ is supposed to be naturally embedded in $R^{d+1}$. Now we construct the desired $(d+1)$-polyhedron. Let $x_{U}$ be the largest $x^{1}$-coordinate of $Z(A)$, and let $x_{L}$ be the smallest $x^{1}$-coordinate of $Z(B)$. Define Shift $=x_{U}-x_{L}$, and

$$
v_{\text {Shift }}=(\text { Shift }+1,0, \ldots, 0) \in R^{d+1} \text {. }
$$

Then $B^{1}=B+v_{\text {Shift }}+e^{d+1}$ is the shifted copy of $B$ in the hyperplane $x^{d+1}=1$.

Consider the polyhedron $C=\operatorname{Conv}\left(A \cup B^{1}\right)$. Note that $Z(C)=Z(A) \cup Z\left(B^{1}\right)$ because $C$ lies entirely in the slab $0 \leq x^{d+1} \leq 1$. Now it is easily seen that the polyhedron $C$ generates the required concatenated string.

Theorem 3. Let $d \geq 2$ and $k \leq 2^{d-1}$. Then each string of nonnegative integers of length $k$ is d-polyhedral.

Proof. By induction. For $d=2$ the statement is evident. If $k \leq 2^{d-2}$, then Theorem 3 holds by the induction hypothesis if we observe that a $d-1$-polyhedral 
string is also a $d$-polyhedral one. Otherwise, if $2^{d-2} \leq k \leq 2^{d-1}$, then $k=k 1+k 2$, where $k 1=2^{d-2}$ and $k 2 \leq 2^{d-2}$. Let us split the original string into two substrings of lengths $k 1$ and $k 2$, respectively. By the induction hypothesis they are both $d-1$-polyhedral, hence the claim of Theorem 3 follows from Lemma 3 .

We may readily suspect that some sufficiently long strings may be nonpolyhedral. (Actually, as we shall see below, the length $2^{d-1}+1$ will do.) However, counterexamples are not so evident as may seem. From the cases $d=2,3$ we might conclude that sufficiently long strings of type

$$
\langle\text { Large 1, 1, 1, ., 1, Large 2〉 }
$$

might be nonpolyhedral. However, this is not the case. In fact, all of them are already 4-polyhedral! Actually, the consecutive substrings $\langle$ Large1 $\rangle,\langle 1,1, \ldots, 1\rangle$, and $\langle$ Large 2$\rangle$ are clearly 2-polyhedral. Now we apply the merging lemma, Lemma 1 , twice to obtain the 3 -polyhedral string $\langle$ Large 1, 1, ., 1〉 first and then the 4-polyhedral realization of

$$
\langle\text { Large1, 1, 1, ., 1, Large } 2\rangle \text {. }
$$

This example, however, gives a hint of the possible structure of an unfavorable string: it is not enough for it to be "thin" inside, it must also be "concave everywhere."

Definition. A function $U(x)$ is defined to be strongly concave in domain $D$ if, for any integer $x 1, x 2$ from $D$,

$$
U((x 1+x 2) / 2)<(U(x 1)+U(x 2)) / 2-1 .
$$

For example, $U_{k}(x)=k^{x}, x \geq 3, k \geq 2$, is strongly concave.

Denote $T=2^{d-1}+1$.

Theorem 4. Let $U(i)$ be an integer strongly concave function on the interval $[1, \ldots, T]$ satisfying the conditions $U(2) \leq U(T-1), U(1)=U(T)>U(T-1)^{*} T$, and $U(i) \geq 2, i=1, \ldots, T$. Then the string

$$
S=\left\langle(U(1))^{d}+1, U(2), U(3), \ldots, U(T-1),(U(T))^{d}+1\right\rangle
$$

is nonpolyhedral for $d>2$.

(For example, function $T^{x}$ satisfies the conditions.)

Proof. Consider the contrary and let $P$ be the polyhedron generating $S$, with its first level being at $x^{1}=1$. By Theorem $2, U(1)$ integer points on a common line can be found in level 1 , the same holds for level $T$. Denote the corresponding line segments by $L_{1}$ and $L_{T}$, respectively. 
(a) First we prove that $L_{T}$ is parallel to $L_{1}$, and, what is more, at each intermediate level of $P$ the integer points lie on a line parallel to $L_{1}$.

Assume the contrary: at level $i, 3 \leq i \leq T$, some two $(U(i) \geq 2$ !) integer points lie on a line not parallel to $L_{1}$. (The case $i=2$ may be considered afterward in a symmetric way, with respect to $L_{T}$.) The shortest segment of this line with endpoints in $Z(P)$ is denoted by $L_{i}$.

Consider the three-dimensional integer sublattice whose basis consists of $e^{1}, l$ (the directional vector of $L_{i}$ ), and $l^{1}$ (the shortest lattice vector of $L_{1}$ ). Consider the projection of the tetrahedron determined by $L_{1}$ and $L_{i}$ on a plane normal to $L_{1}$. The area of the resulting triangle (measured in the basis $\left\{e^{1}, i\right\}$ ) is not less than one unit (because we have taken $i \geq 3$ ). Therefore, this triangle contains at least one lattice point at level $i-1$ of $P$. Hence, in the tetrahedron this point corresponds to a segment in direction $l^{1}$ of length at least

$$
\left|L_{1}\right| / i \geq\left|L_{1}\right| / T=U(1) / T>U(2)
$$

lattice units. However, by strong concavity of function $U$ and the conditions of Theorem 4 no intermediate level of $P$ may contain more than $U(2)$ integer points.

(b) Now we know that at all levels of $P$, integer points must lie on lines parallel to $L_{1}$. Denote the corresponding line segments similarly by $L_{2}, \ldots, L_{T-1}$. Consider the projection $M: R^{d} \rightarrow H$, where $\mathrm{H}$ is a hyperplane normal to all these $L_{1}$. This projection maps the lattice $Z^{d}$ onto a lattice $Z_{H}$ in $H$. The segments $L_{i}$ are mapped into the points $Q_{i}$ of $Z_{H}$.

As in the proof of Theorem 1 , among $T=2^{d+1}+1$ points $\left\{Q_{i}\right\}$ in a $(d-1)$ dimensional lattice $Z_{H}$ there exist two points $Q_{k}$ and $Q_{m}$ such that $Q=\left(Q_{k}+Q_{m}\right) / 2 \in Z_{H}$. The lines $M^{-1}\left(Q_{k}\right)$ and $M^{-1}\left(Q_{m}\right)$ contain $U(k)$ and $U(m)$ integer points of $P$, respectively. By convexity, $M^{-1}(Q)$ must contain at least $(U(k)+U(m)) / 2-1$ integer points of $P$, but this contradicts the strong concavity of $U$, hence, Theorem 4 is proven.

\section{References}

[GW] Gritzmann, P., and J. M. Wills. Lattice points. In Handbook of Convex Geometry, P. M. Gruber and J. M. Wills, eds. North-Holland, Amsterdam, to appear.

[KM] Korneenko, N. M., and N. N. Metelskij. Description of partial orders of feasible solutions of integer linear programs, Dokl. Akad. Nauk BSSR, 22(3) (1978), 208-211 (in Russian).

[M] Metelskij, N. N. On the equivalence of discrete optimization problems, Vestsi Akad. Navuk BSSR Ser. Fiz.-Mat. Navuk, No. 1 (1973), 5-9 (in Russian).

[S] Schrijver, A. Theory of Linear and Integer Programming. Wiley, New York, 1986.

Received March 20, 1992. 